\title{
Adaptación al cambio climático y la gestión del riesgo en comunidades del río Tempisque, Costa Rica
}

Adaptation to Climate Change and Risk Management in Communities in the Tempisque River, Costa Rica

Adaptação à mudança climática e gestão de risco em comunidades do Rio Tempisque, Costa Rica

\author{
Marcela Gutiérrez Miranda \\ Universidad Nacional de Costa Rica. Heredia, Costa Rica \\ marcela.gutierrez.miranda@una.cr \\ https://orcid.org/0000-0001-6300-8251 \\ María Carolina Fernández Saborío \\ Universidad de Costa Rica. San José, Costa Rica \\ maria.fernandezsaborio@ucr.ac.cr \\ https://orcid.org/0000-0002-9117-1336 \\ María José Valverde Barquero \\ Universidad Nacional de Costa Rica. Heredia, Costa Rica \\ maria.valverde.barquero@est.ac.cr \\ https://orcid.org/0000-0002-8175-4028
}

DOI: https://doi.org/10.32719/25506641.2021.10.4

Presentado: 16 de octubre de 2020 - Revisado: 13 de noviembre de 2020

Aceptado: 26 de enero de $2021 \cdot$ Publicado: 1 de julio de 2021

Artículo de investigación

Licencia Creative Commons

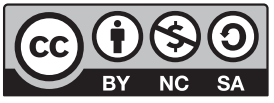




\section{Resumen}

Se analizan las acciones realizadas desde la academia para contribuir al mejoramiento de la gestión local, mediante la implementación de mecanismos de adaptación al cambio climático y la gestión de riesgos en tres comunidades de la cuenca baja del río Tempisque en Costa Rica. El proyecto utiliza como metodología la investigación acción participativa, involucrando a los diversos actores y generando cambios en sus prácticas.

El principal resultado muestra que las acciones ejecutadas en educación ambiental, campañas de recolección de residuos, reforestación y turismo rural, han contribuido a aumentar el sentido de ciudad y preservación del medioambiente en las personas que habitan la comunidad, a través del entendimiento de la relación que existe entre la conservación del ecosistema y la mejora de las condiciones de vida en las comunidades. Además, se evidencia cómo la variabilidad climática incide en el desarrollo económico ya que afecta la capacidad productiva de la zona y cómo el abordaje multidisciplinario del proyecto incide de manera directa sobre la conservación del ecosistema y el desarrollo de las comunidades.

Palabras clave: adaptación al cambio climático, prevención de desastres, resiliencia, comunidad, desarrollo comunitario.

JEL: Q57 Economía ecológica, servicios del ecosistema, conservación de la biodiversidad, bioeconomía.

\section{Abstract}

The actions carried out by the academy are analyzed to contribute to local management improvement through the implementation of climate change adaptation mechanisms and risk management in three communities in the Lower Tempisque River Basin in Costa Roca. The project uses participatory action research as a methodology, involving the various actors and generating changes in them.

The main result shows that the actions carried out in: environmental education, waste collection campaigns, reforestation, rural tourism, among others, have contributed to increasing the sense of care and environment preservation in the people of the community, through the understanding of the relationship that exists between the ecosystem conservation and the living conditions improvement in the communities. In addition, it is evident how climate variability affects economic development since it affects the productive capacity area and how the project multidisciplinary approach directly affects the ecosystem conservation and the communities' development.

Keywords: adaptation to climate change, disaster prevention, resilience, community, community development.

JEL: Q57 Ecological Economics, Ecosystem Services, Biodiversity Conservation, Bioeconomics. 


\section{Resumo}

Analisam-se as ações realizadas a partir da academia para contribuir para a melhoria da gestão local por meio da implementação de mecanismos de adaptação à mudança climática e a gestão de riscos em três comunidades da Bacia Inferior do Rio Tempisque, na Costa Rica. O projeto utiliza como metodologia a pesquisa de ação participativa, envolvendo diversos atores e gerando mudanças em suas práticas.

O principal resultado mostra que as ações executadas em: educação, ambiente, campanhas para coleta de resíduos, reflorestamento e turismo rural, entre outras, contribuíram para aumentar o sentido de cuidado e preservação do meio ambiente nos habitantes da comunidade por meio do entendimento da relação entre a conservação do ecossistema e a melhoria das condições de vida nas comunidades. Além disso, evidencia-se como a variabilidade climática incide sobre o desenvolvimento econômico, uma vez que afeta a capacidade produtiva da região, e como a abordagem multidisciplinar do projeto incide de maneira direta sobre a conservação do ecossistema e sobre o desenvolvimento das comunidades.

Palavras-chave: adaptação à mudança climática, prevenção de desastres, resiliência, comunidade, desenvolvimento comunitário.

JEL: Q57 Economia ecológica, serviços ecossistêmicos, conservação da biodiversidade, bioeconomia.

\section{Introducción}

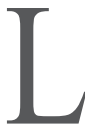

as comunidades poseen una relación intrínseca con el ecosistema en el que habitan, pueden tanto contribuir a su desarrollo y conservación como verse limitadas por los bienes y servicios que el hábitat les provee. Los seres humanos pueden obtener de estos elementos fundamentales medios para su subsistencia así como recursos que les permitan desarrollarse económicamente.

No obstante, ante la realidad latente del cambio climático, se ven afectados los ecosistemas y por lo tanto las comunidades; sequías, erosión, lluvias torrenciales y cambios de temperatura, son factores que afectan las formas en que las actividades agropecuarias y la vida de las personas se desenvuelven.

Ante esto nace HuriTe, una iniciativa conjunta de carácter interdisciplinario entre la Universidad Nacional de Costa Rica (UNA), desde su Centro de Estudios Generales (CEG), y la Universidad de Costa Rica (UCR), desde la Escuela de Administración Pública (EAP), cuyo objetivo es contribuir al 
mejoramiento de la gestión local por medio del desarrollo de capacidades productivas, sociales y organizativas en las poblaciones de la cuenca baja del río Tempisque, en Costa Rica (CBRT), como mecanismo de adaptación a los efectos de la variabilidad del clima y su impacto en estos ecosistemas (CR Consejo Nacional de Rectores 2017).

Esta investigación visibiliza los proyectos de gestión y desarrollo de capacidades productivas en comunidades afectadas por el cambio climático a través del proyecto HuriTe que integra las necesidades ambientales con las realidades de las personas y busca generar respuestas a través de la educación ambiental, la gestión del riesgo ante desastres y la gestión socioeconómica.

\section{Conceptualización}

Los ecosistemas son aquellas unidades funcionales conformadas por seres vivos que interactúan entre sí y con el ambiente en un espacio definido (ONU 2005). Los servicios ecosistémicos son los recursos naturales que proveen al ser humano, y a las demás especies, de bienes y servicios necesarios para su subsistencia. Las personas son parte integral los ecosistemas y sus servicios y requieren de su integridad para sobrevivir: suelos que produzcan alimentos de calidad, estabilidad climática y ecosistemas saludables que brinden condiciones permitiendo el desarrollo y el bienestar de la sociedad.

Actualmente, uno de los factores que más afecta a los ecosistemas, y consecuentemente al ser humano es el cambio climático. Esto expone al ser humano a condiciones de mayor vulnerabilidad, afecta los medios de subsistencia, los ingresos, la migración local y ocasiona conflictos sociopolíticos. Asimismo, impacta en la seguridad económica y física, la libertad, las relaciones, la salud, el bienestar, la calidad de vida y el desarrollo de las comunidades y de los individuos (CR Sistema Nacional de Áreas de Conservación de Costa Rica - SINAC- 2020).

El cambio climático (en adelante CC) es definido como el "cambio de clima atribuido directa o indirectamente a la actividad humana que altera la composición de la atmósfera global y que se suma a la variabilidad natural del clima observada durante períodos de tiempo comparables" (ONU 1992, 3). 
A nivel mundial, existe un consenso científico sobre el impacto del cambio climático como resultado del aumento de los gases de efecto invernadero (GEI) tales como el dióxido de carbono, metano, óxidos nitrosos, clorofluorocarbonos, entre otros, en la atmósfera sobre el planeta y su respectiva dinámica. Esto ha provocado aumentos en la temperatura del planeta y como respuesta a este calentamiento global (causa probable), se prevén cambios que afectan - y afectarán — a todas las especies que habitan el planeta: aumento del nivel del mar, aumento de la temperatura global, calentamiento de los océanos, disminución de las placas de hielo y casquetes polares, retroceso de los glaciares, eventos hidrometeorológicos extremos, y acidificación de los océanos (IPCC 2019). Sin embargo, los pobladores de las costas y los que dependen del recurso marino costero son más susceptibles a situaciones de riesgo. En las zonas costeras se ubica mayormente el desarrollo económico, debido a la industria hotelera, puertos, zonas agrícolas, y las variaciones climáticas, sequías, huracanes y tormentas devastan primeramente dichas zonas (Grupo Laera y Global Climate Adaptation Partnership 2014).

De acuerdo con Stern (2006), el cambio climático afectará los elementos básicos de la vida de los seres humanos, la flora y fauna, acceso al agua, la producción de alimentos, la salud y el medioambiente, elementos que pueden llegar a verse comprometidos con una población que los demanda de forma permanente. Cientos de millones de personas podrían sufrir hambre, escasez de agua e inundaciones conforme el mundo se calienta y se enfría de forma extrema. Actuar de manera anticipada ante el cambio climático es crucial y en esas condiciones los costos son muy bajos comparados con el de no hacer nada.

Amparado en el tema del cc y el aumento de sus consecuencias, la actual concepción de gestión de riesgo pone énfasis no solo en la atención de emergencias, sino en la prevención. En Costa Rica se define a la gestión de riesgo como un modelo que incorpora criterios de preparación, atención y recuperación de emergencias, así como la prevención y mitigación de desastres y revertir las condiciones de vulnerabilidad (CR Comisión Nacional de Prevención de Riesgos y Atención de Emergencias - CNE - 2015), teniendo en cuenta que desde la teoría social del riesgo, la vulnerabilidad está definida por las condiciones sociales, económicas, culturales o instituciona- 
les relacionadas con el grado de exposición de un grupo a los efectos de una amenaza (Andrade y Laporta 2009).

Aunado a este concepto y su asociación con el riesgo climático, el PNUD (2010) establece que la gestión de riesgo climático se centra en la adaptación de los sectores como la agricultura, los recursos hídricos o la seguridad alimentaria que son altamente sensibles a la variabilidad del clima y que influyen directamente en el bienestar de las personas. Por lo tanto, el replanteamiento del desarrollo debe de estar acompañado por el fortalecimiento de las capacidades que permitan una gestión de riesgo intersectorial (Oficina de las Naciones Unidas para la Reducción del Riesgo de Desastres - UNSOR2015). Esto implica generar la capacidad de uso de los ecosistemas mediante prácticas innovadoras para gestionar las acciones de desarrollo, ser inclusivos en la participación ciudadana local y direccionar hacia una sociedad más resiliente.

Las comunidades deben ser capaces de hacer frente a los eventos adversos y ante esto surge la resiliencia como un elemento clave en la gestión del riesgo. La UNISDR $(2009,28)$ define a la resiliencia como "la capacidad de un sistema, comunidad o sociedad expuestos a una amenaza para resistir, absorber, adaptarse y recuperarse de sus efectos de manera oportuna y eficaz, lo que incluye la preservación y la restauración de sus estructuras y funciones básicas". En Costa Rica, la política nacional de gestión de riesgos incluye la capacidad de ser transformados positivamente por los eventos adversos, generando cambios a las comunidades, esto con el fin de adaptarse y basarse en las nuevas oportunidades que pueden llegar a surgir. Es necesario un ajuste de los sistemas naturales o humanos como respuesta a los estímulos climáticos actuales o esperados (IPCC 2007).

\section{Cambio climático y gestión del riesgo en Costa Rica}

Costa Rica se ve afectada por las variaciones en el clima, fenómeno que ha ocasionado cambios globales que comprometen el bienestar y los medios de vida de los seres humanos. Los factores geográficos como la ubicación y su tamaño son elementos clave que determinan su vulnerabilidad ante las consecuencias del calentamiento global. A nivel nacional se han experimen- 
tado los dos períodos más calurosos en los últimos diez años, según los registros: 2005 y 2010 (CR MINAE 2018b). Además, las zonas de alto riesgo son las partes altas de las montañas, los manglares, los humedales, los arrecifes y los bosques en zonas calientes como la bajura de las costas atlántica y pacífica (CR MINAE 2018b).

Las pérdidas generadas por fenómenos hidrometeorológicos en los sectores de infraestructura, recursos hídricos, agropecuario, servicios públicos, biodiversidad y el ámbito privado, están en aumento. La CNE del país estimó que solo las pérdidas totales ocasionadas por la tormenta tropical Nate en 2017 equivalen al 1\% del Producto Interno Bruto Anual de Costa Rica (CR MINAE 2018a).

Una de las zonas de afectación es la provincia de Guanacaste la cual se encuentra ubicada en la región Chorotega en el Pacífico norte (Retana y Calvo 2018). En los últimos años, en esta zona la frecuencia y afectación de eventos hidrometeorológicos se han incrementado lo que agudiza su vulnerabilidad. El SINAC (2016) establece como impactos del cambio climático la disminución de los caudales, un aumento en las temperaturas y en el nivel del mar que provoca erosión en las playas, reducción en los niveles freáticos, cambios en los patrones de lluvias y afectaciones sobre los sistemas productivos.

De acuerdo con Vargas y Gómez (2017), la región Chorotega, específicamente la cuenca baja del río Tempisque, es uno de los sitios más afectados por el calentamiento global en el país. Los cambios en los patrones de precipitación han originado que las temporadas de lluvia sean muy cortas, lo que ha generado sequías, aumento en las temperaturas y aridez. El caudal del principal río de la provincia, el Tempisque, y de sus afluentes, disminuye considerablemente en la época seca, repercutiendo en la disponibilidad de agua para el riego de los diferentes cultivos de la zona tales como: arroz, café, maíz, frijoles, caña de azúcar, raíces tropicales (tiquisque, yuca, ñampí y ñame), sandía, papaya, melón, pasto para ganadería de carne y leche, y hortalizas. Lo anterior genera que la capacidad productiva de los suelos guanacastecos se vea comprometida.

Además, Hartley (2012) afirma que en la provincia las precipitaciones pluviales han experimentado una disminución del $25 \%$ ocasionando sequías agrícolas que se manifiestan cuando el suelo pierde la humedad suficiente 
para garantizar el crecimiento de los cultivos. Este tipo de sequía ha afectado a los suelos de todos los cantones de la región. Según datos del Ministerio de Agricultura y Ganadería de Costa Rica (2018) de 2005 al 2011 las pérdidas en el sector agropecuario en Guanacaste representaron el 25,2\% del monto total a nivel nacional, lo que la sitúa como la segunda provincia con mayor cantidad de pérdidas debido a fenómenos hidrometeorológicos y refleja que las variaciones climáticas afectan factores económicos, impactan las condiciones de vida de las personas e inciden en el aumento de la vulnerabilidad.

\section{Políticas y marco normativo}

Ante un entorno cada día más afectado por el cambio climático, es indispensable contar con un marco normativo robusto que permita responder de manera adecuada y anticipada a los riesgos que se puedan presentar. Ante esto, en materia legal, Costa Rica ha logrado migrar de la conceptualización tradicional de la gestión de riesgo a gestión de desastres, a un concepto más amplio que además implica su prevención y reducción de riesgos a desastres.

Lo anterior se visualiza en el marco legal en materia de gestión de riesgos, específicamente en la Ley No. 8488, Ley Nacional de Emergencias y Prevención que tiene como finalidad "la reducción de las causas del riesgo, así como el manejo oportuno, coordinado y eficiente a situaciones de emergencia" (CR Asamblea Legislativa 2006, art. 2). En esta se define dicho concepto como un proceso que permite prevenir y mitigar las condiciones de vulnerabilidad.

Asimismo, en respuesta a la Tercera Conferencia Mundial de las Naciones Unidas y el Marco de Acción de Sendai para la Reducción del Riesgo de Desastres 2015-2030, en donde se expresa la necesidad de comprender todas las dimensiones (UNISDR 2020); se establece en Costa Rica la Política Nacional de Gestión del Riesgo 2016-2030 que comprende factores subyacentes como lo son los elementos económicos y sociales (CNE 2015). Esta política sienta las bases conceptuales y los lineamientos CR para orientar, por 15 años, la elaboración sucesiva del Plan Nacional de Gestión de Riesgo en períodos quinquenales con fecha de finalización en 2030 y establece la 
forma en la que se deberá organizar la ejecución, asegurar la fiscalización y medir los resultados.

Sin embargo, a pesar de que Costa Rica cuenta con una política que le permite gestionar las amenazas meteorológicas, cada día son mayores las afectaciones ocasionadas por el cambio climático que "posiblemente es la amenaza más relevante que afrontaremos como sociedad" (Mora citado en MINAE 2018, 11), por lo cual es indispensable no solo organizar los riesgos, sino también adaptarse a ellos.

Son múltiples los documentos, planes, agendas, y convenciones - nacionales e internacionales - que sirven como base para la creación de la Política Nacional de Adaptación al Cambio Climático 2018-2030: la Estrategia Nacional de Cambio Climático 2009-2012, la Agenda 2030 para el Desarrollo Sostenible, el Pacto Nacional para el Avance de los ODS; el Plan Nacional de Desarrollo 2015-2018, la Convención Marco de las Naciones Unidas sobre el Cambio Climático 2015, el Acuerdo de París que fue ratificado a nivel nacional por la Asamblea Legislativa en 2016, entre otros. La Política Nacional de Adaptación al Cambio Climático 2018-2030 busca "fortalecer capacidad adaptativa y condiciones de resiliencia; reducir la vulnerabilidad, moderar daños materiales y evitar pérdidas humanas, así como aprovechar las oportunidades que se presentan detrás de cada amenaza" (MINAE 2018, 16). Para ello, se establece un marco de orientación estratégico para la planificación del desarrollo, y se espera crear un sistema de corresponsabilidad que permita orientar las inversiones públicas y privadas para reducir el impacto del cambio climático. Lo anterior busca garantizar lo establecido en la Constitución Política de Costa Rica: "Toda persona tiene derecho a un ambiente sano y ecológicamente equilibrado" (CR Asamblea Nacional Constituyente 1994, art. 50), y el art. 50 de la Ley, No. 7554 Ley Orgánica del Ambiente: “El agua es de dominio público, su conservación y uso sostenible son de interés social" (CR Asamblea Legislativa 1994, art. 50). Recientemente, mediante reforma a la Constitución Política Nacional se estableció el derecho al agua como un derecho humano.

Adicionalmente, en 2017 se publicó la Política Nacional de Humedales, para direccionar la protección de estos importantes ecosistemas que son altamente vulnerables a la crisis climática (PNUD 2018). Y en 2019 se publicó el Plan Nacional de Desarrollo y de Inversión Pública del Bicentenario 
2019-2022 de Costa Rica, que establece en sus metas lograr la descarbonización de la economía (CR MIDEPLAN 2019) a esto se suman programas de reducción de emisiones y certificaciones en diferentes temas productivos que pretenden orientar el accionar de las organizaciones y promover hábitos organizacionales compatibles con la conservación del medioambiente.

\section{Respuesta integral: una solución basada en comunidad}

\section{Contexto: escenario universitario}

La universidad pública costarricense es un espacio de libertad y democracia, que ha estado comprometida con el bienestar de la sociedad a través del desarrollo de sus actividades sustantivas como lo son la docencia, la investigación, la producción y la extensión-acción social (CR Consejo Nacional de Rectores 2016).

El Consejo Nacional de Rectores (CONARE) es un órgano establecido producto del "Convenio de Coordinación de la Educación Superior Universitaria Estatal en Costa Rica" (CR Consejo Universitario 1987), suscrito por las instituciones de educación superior universitarias estatales del país.

El Sistema de Educación Superior Universitaria Estatal Costarricense, propicia la integración y articulación entre las universidades que lo conforman y tiene como objetivo: generar oportunidades para el desarrollo del país por medio de la democratización y del conocimiento para favorecer la ampliación y creación de nuevas alternativas de desarrollo social, cultural, económico, ambiental y científico (CONARE 2016). Para cumplir con este objetivo, el CONARE financia acciones interuniversitarias para el fortalecimiento de la educación superior estatal. Las iniciativas deben impulsar acciones y tareas de construcción y desarrollo del sistema de educación superior universitaria estatal y enmarcarse en el contexto del Plan Nacional de la Educación Superior (PLANES) vigente al momento de la formulación (CONARE 2019).

Es así como, en 2018 inicia el proyecto "Desarrollo de un modelo de gestión local integral con las poblaciones de los humedales de la cuenca baja del río Tempisque" (figura 1) en el eje de extensión y acción social, bajo el 
objetivo estratégico de contribuir con la transformación de la realidad social mediante actividades concertadas y articuladas con las comunidades, que propicien el intercambio de experiencias y la construcción conjunta de soluciones a los problemas que enfrentan (CONARE 2017).

\section{Figura 1}

Mapa de los humedales de la cuenca baja el río Tempisque

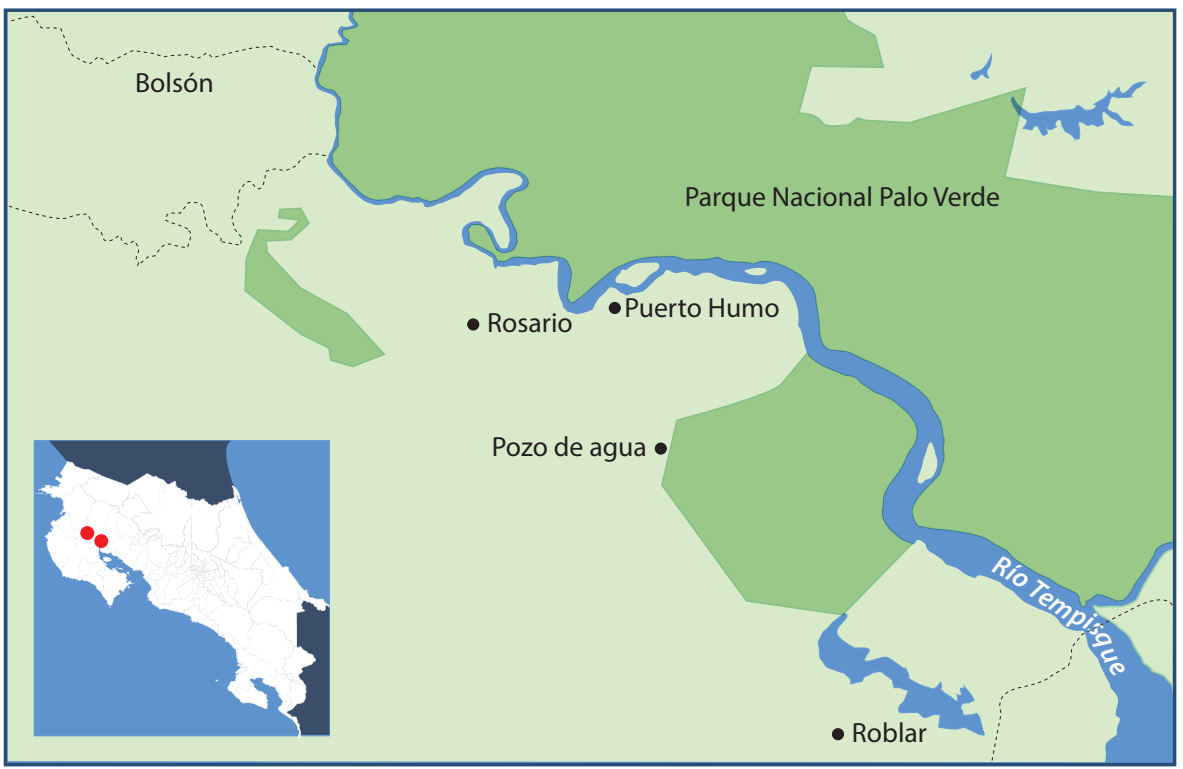

Fuente: María José Elizondo (2020).

\section{Metodología}

Para el análisis de las acciones realizadas, desde la academia, a través del proyecto HuriTe se realizó una investigación cualitativa con un foco de interés amplio. El diseño utilizado es etnográfico ya que el mismo permitió conocer el grupo humano beneficiario, sus experiencias, actitudes y reflexiones, para así conocer el impacto del proyecto en las comunidades (Villalobos 2019). Como técnicas de recolección de información, se realizó una revi- 
sión bibliográfica en los temas centrales del proyecto, además se realizaron entrevistas a profundidad con los académicos involucrados y se realizaron dos grupos focales con los beneficiarios del proyecto. La información recolectada a través de dichas técnicas fue sistematizada y analizada para la identificación de los elementos que podrían generar mayor impacto en las comunidades.

El enfoque metodológico que se utiliza es el de la investigación acción participativa (IAP) como elemento de transformación social (Colmenares 2011). El diagnóstico participativo permitió identificar la realidad y necesidades de las comunidades; posteriormente, se construyó y ejecutó un plan de acción en los temas del proyecto. Además, se realizó una aproximación desde la teoría social del riesgo y sus dimensiones: amenaza, vulnerabilidad exposición e incertidumbre (Andrade y Laporta 2009). Durante todo el proceso de ejecución de la iniciativa, la reflexión continua con la participación de todos los involucrados ha sido una constante; lo que permite el análisis, replanteamiento y la reorganización de las acciones en respuesta a los cambios por parte de los involucrados en la investigación, y facilita redimensionar nuevas acciones en atención a las reflexiones realizadas.

\section{Comunidades de la cuenca baja del río Tempisque (CMRT)}

Las comunidades de Rosario, Puerto Humo y Pozo de Agua, se encuentran ubicadas en el distrito de San Antonio, cantón Nicoya, provincia de Guanacaste, Costa Rica. Este distrito posee una población estimada de 6642 habitantes (CR Instituto Nacional de Censos 2002) y, según el Índice de Desarrollo Social de Costa Rica (IDS) se encuentra en la posición 321 de 483 distritos en total (CR MIDEPLAN 2017). Esto evidencia que los habitantes de la zona presentan dificultades para acceder a recursos y servicios lo que afecta su vulnerabilidad.

Estas comunidades se ubican en el margen izquierdo del río Tempisque que desemboca en el océano Pacífico y en su cuenca media y baja alimenta múltiples humedales. Estos ecosistemas acuáticos son estacionales y algunos desaparecen por completo durante la época seca. Por la riqueza biológica, la 
belleza escénica y los múltiples servicios ecosistémicos que brindan estos territorios, se han establecido áreas silvestres protegidas donde destaca el sitio Ramsar Palo Verde que comprende los humedales Parque Nacional Palo Verde (PNPV), Reserva Biológica Lomas Barbudal, los Refugios de Vida Silvestre Mata Redonda, Cipancí y el Tendal; y los humedales Sonzapote, Corral de Piedra, laguna Madrigal y Zapandí (Murillo y Miranda 2018), comunidades de la CBRT beneficiarias del proyecto. Las comunidades donde se ha desarrollado el proyecto son:

Rosario de Nicoya: comunidad rural que tiene una población estimada de 371 habitantes. Se encuentra el Refugio Nacional de Vida Silvestre Mata Redonda donde se ubica la Laguna Mata Redonda que alberga múltiples aves residentes y migratorias. Rosario limita al norte con el Parque Nacional Palo Verde, al este con Puerto Humo, al sur con el cantón Nicoya y al oeste con Bolsón (CR Universidad Nacional de Costa Rica 2018).

Puerto Humo: limita al norte y al este con el río Tempisque, al sur con la localidad de Pozo de Agua y al oeste con Rosario. Es una comunidad pequeña donde viven actualmente 210 habitantes. A principios del siglo pasado fue un importante puerto fluvial que representaba una de las puertas de acceso de bienes a la provincia de Guanacaste. Sin embargo, el desarrollo de nuevas carreteras y los cambios en los medios de transporte han ocasionado que en la actualidad enfrente condiciones de abandono institucional, infraestructura deficiente y pobreza (Cortés, comunicación personal).

Pozo de Agua: limita al norte con Puerto Humo, al este con el río Tempisque, al sur con la comunidad de Roblar y al oeste con Piedras Blancas. Esta se ubica a orillas del humedal Corral de Piedra (Área Silvestre protegida que se encuentra mayoritariamente conformada por propiedades privadas). Este humedal se caracteriza por ser una zona de terrenos planos con lagunas interconectadas, sujeta a inundaciones estacionales de gran magnitud. Es un refugio, alimentación y reproducción para aves y mamíferos. Adicionalmente, funciona como un área de amortiguamiento durante las crecidas del río Tempisque en la época lluviosa previniendo inundaciones. Enfrenta problemáticas relacionadas con la cacería y pesca, así como la construcción ilegal de canales, incendios forestales y pérdida de espejos de agua por la proliferación de la tifa (Thypha domingensis). 
Estas tres comunidades, ubicadas en la CBRT, comparten características socioculturales y ambientales muy similares. Las que se encuentran asentadas en la bajura guanacasteca poseen vestigios de bosque tropical seco y en el período seco se caracterizan por sus altas temperaturas con una duración promedio de cinco meses entre diciembre a abril, mientras que el período lluvioso se extiende de mayo a noviembre (CR Universidad Nacional de Costa Rica 2018).

Durante todo el año la zona presenta altas temperaturas, lo que condiciona, en gran medida, los estilos de vida de las personas que habitan en estos territorios. Debido a las condiciones del clima, las actividades laborales y el trabajo de campo se realizan desde horas tempranas para evitar el desgaste físico causado por el calor. En la época lluviosa, los caminos se deterioran porque se estanca el agua lo que dificulta la movilidad de las personas entre comunidades, propicia la proliferación de vectores y limita el intercambio comercial en la zona (Ureña, comunicación personal).

Los pobladores de estas comunidades dependen para su subsistencia de la conservación de los humedales (río Tempisque, Refugio Nacional de Vida Silvestre Mata Redonda y humedal Corral de Piedra). Sin embargo, factores como el calentamiento global — que está provocando el secado de las zonas de humedales - , la deforestación, el uso excesivo de agroquímicos cuenca arriba y prácticas extractivas de sobreexplotación, comprometen, de manera alarmante, la salud del ecosistema y el bienestar de esta población. Además, se suma por mucho tiempo una subvaloración de estos territorios por parte del Estado costarricense lo que propició su deterioro.

La cercanía con los humedales, el clima de la zona, su topografía, su geografía, así como los hábitos y prácticas de vida de sus pobladores son elementos que influyen en las afectaciones que tienen los habitantes de estas localidades. La tabla 1 presenta las amenazas que enfrentan las comunidades de la CBRT y los diferentes grados de vulnerabilidad y exposición al riesgo que presentan. 
Tabla 1

Amenazas que enfrentan las comunidades de la cuenca baja del río Tempisque

\begin{tabular}{|l|l|}
\hline \multicolumn{1}{|c|}{ Amenaza } & \multicolumn{1}{c|}{ Exposición-Vulnerabilidad } \\
\hline Inundaciones y flujos de lodo & $\begin{array}{l}\text { El río Tempisque es caudaloso y a su paso recibe múltiples } \\
\text { afluentes que depositan sedimentos, residuos y otros materiales } \\
\text { en su cauce. Las comunidades se asentaron en las planicies del } \\
\text { río y su desarrollo urbanístico y agropecuario está marcado por } \\
\text { la poca planificación territorial. }\end{array}$ \\
\hline Sismos & $\begin{array}{l}\text { El cantón de Nicoya se encuentra dentro de una región sísmica } \\
\text { caracterizada por presentar eventos generados por el choque } \\
\text { de placas Coco-Caribe. }\end{array}$ \\
\hline Inestabilidad y saturación de & $\begin{array}{l}\text { La topografía de la zona provoca inestabilidad y rápida satura- } \\
\text { ción de los suelos. Las intensas lluvias provocan deslizamientos } \\
\text { e inundaciones con daños a infraestructura vial, al ganado y a } \\
\text { los cultivos. }\end{array}$ \\
\hline Fenómeno del Niño & $\begin{array}{l}\text { La municipalidad de Nicoya no brinda un servicio regular de re- } \\
\text { colección de residuos sólidos en estos territorios de la CBRT. } \\
\text { Además, la gestión integral de los residuos que realiza este go- } \\
\text { bierno local es débil y depende en gran medida de iniciativas } \\
\text { comunales y de otras instituciones con presencia en la zona } \\
\text { (SINAC, centros educativos, universidades, asociaciones de } \\
\text { desarrollo entre otros). Usualmente, los vecinos eliminan sus } \\
\text { residuos en sitios clandestinos (botaderos a cielo abierto), los } \\
\text { entierran ylo queman. }\end{array}$ \\
\hline Manejo de residuos sólidos \\
\hline $\begin{array}{l}\text { Según el Instituto Meteorológico de Costa Rica, el fenómeno } \\
\text { El Niño se refiere a la interacción climática océano-atmósfera } \\
\text { a gran escala, asociada a un calentamiento periódico (que es } \\
\text { recurrente) de las temperaturas de la superficie del mar, exten- } \\
\text { diéndose en todo el océano Pacífico ecuatorial central y el Pa- } \\
\text { cífico este-central. Este fenómeno ocurre de forma periódica y } \\
\text { provoca sequías prolongadas. }\end{array}$ \\
\hline
\end{tabular}

Fuente: PNUD y MIDEPLAN (2014). 
Las comunidades de la CBRT enfrentan múltiples amenazas que podrían afectar el bienestar de la población y su desarrollo. Por lo tanto, es necesario implementar medidas que permitan a la población adaptarse para mitigar los posibles efectos de los diversos fenómenos y/o condiciones. Para lograrlo, es necesario que se realice un trabajo intersectorial y multidisciplinario que involucre, desde el inicio, a los pobladores de la región y a las diversas instituciones. Fue a partir de esta realidad que el proyecto HuriTe desarrolló, en conjunto con el SINAC y la sociedad civil, diversas acciones para adaptarse a las variables condiciones del clima que prevalecen en esta zona del país.

\section{Acciones HuriTe: acciones sociales, económicas y de conservación}

HuriTe ha desarrollado acciones dirigidas a mejorar el tejido organizacional y las condiciones socioeconómicas de los pobladores de la CBRT. Simultáneamente, también se implementaron estrategias para la conservación, restauración y uso sostenible de los ecosistemas como parte de la implementación de un modelo de gestión local integral con las poblaciones de los humedales de esta zona del pacífico costarricense. Las condiciones de riesgo y las amenazas que enfrentan los habitantes de la zona fueron los elementos clave para generar iniciativas innovadoras para la adaptación de las localidades a la variabilidad del clima y sus consecuencias. La tabla 2 detalla las actividades realizadas, la comunidad y el impacto de estas actividades sobre los pobladores.

Las actividades realizadas fueron exitosas porque responden a las necesidades y los vacíos identificados a través del análisis académico y del intercambio con los pobladores. Las acciones son producto de un trabajo participativo, intersectorial y transdisciplinario que involucró a los pobladores, grupos organizados de la sociedad civil, gobierno local, SINAC y actores públicos, liderados por el proyecto HuriTe. En su conjunto, esta iniciativa permitió mejorar el entorno en que viven los pobladores y sus condiciones biofísicas para asegurar la disponibilidad de los diversos servicios ecosistémicos que reciben como elementos clave para su progreso y la sostenibilidad de las comunidades. 
Tabla 2

\section{Resultados de las actividades del proyecto HuriTe en las comunidades de la cuenca baja del río Tempisque}

\begin{tabular}{|c|c|c|}
\hline Actividad & Comunidad & Impacto \\
\hline $\begin{array}{l}\text { Educación ambiental con escolares } \\
\text { (gestión de residuos sólidos, servicios } \\
\text { ecosistémicos del humedal, cambio cli- } \\
\text { mático, gestión de riesgo, Bandera Azul } \\
\text { Ecológica). }\end{array}$ & $\begin{array}{l}\text { Rosario } \\
\text { Pozo de Agua }\end{array}$ & $\begin{array}{l}\text { Mejorar los conocimientos y las } \\
\text { prácticas ambientales de los niños } \\
\text { en las temáticas del programa de } \\
\text { educación ambiental. }\end{array}$ \\
\hline $\begin{array}{l}\text { Campañas interinstitucionales de reco- } \\
\text { lección de residuos sólidos y sensibiliza- } \\
\text { ción en la gestión integral de los residuos } \\
\text { sólidos. }\end{array}$ & $\begin{array}{l}\text { Rosario } \\
\text { Pozo de Agua } \\
\text { Puerto Humo }\end{array}$ & $\begin{array}{l}\text { Comunidades más limpias y sen- } \\
\text { sibilizadas en la gestión integral } \\
\text { de los residuos sólidos. }\end{array}$ \\
\hline Reforestación & $\begin{array}{l}\text { Laguna } \\
\text { Mata Redonda }\end{array}$ & $\begin{array}{l}\text { Mantenimiento de } 215 \text { árboles } \\
\text { después del involucramiento de la } \\
\text { academia y la comunidad. } \\
215 \text { árboles de los sembrados } \\
\text { por el Proyecto Humedales del } \\
\text { SINAC-PNUD } 2017 \text { sobrevivieron } \\
\text { gracias a las labores de riego, } \\
\text { abono y corte de maleza. }\end{array}$ \\
\hline Proyecto de turismo rural comunitario & $\begin{array}{l}\text { Rosario } \\
\text { de Nicoya }\end{array}$ & $\begin{array}{l}\text { Grupo organizado que brinda ser- } \\
\text { vicios de turismo rural comunitario } \\
\text { y beneficia a } 16 \text { familias de la co- } \\
\text { munidad. } \\
\text { La disponibilidad de nuevos ingre- } \\
\text { sos económicos que dependen de } \\
\text { la conservación del ecosistema } \\
\text { despierta en los pobladores un } \\
\text { mayor sentido de cuidado y pre- } \\
\text { servación del medioambiente. }\end{array}$ \\
\hline $\begin{array}{l}\text { Conformación y capacitación del Comité } \\
\text { Local de Gestión de Riesgos. }\end{array}$ & $\begin{array}{l}\text { Rosario } \\
\text { Puerto Humo } \\
\text { Pozo de Agua }\end{array}$ & $\begin{array}{l}\text { Un grupo de vecinos capacitado } \\
\text { para llevar a cabo la primera res- } \\
\text { puesta en sus comunidades ante } \\
\text { una amenaza o un evento catas- } \\
\text { trófico. }\end{array}$ \\
\hline
\end{tabular}

Elaboración propia con datos del proyecto HuriTe (2020). 


\section{Conclusiones y recomendaciones}

La región de Guanacaste, Costa Rica, es altamente vulnerable a las consecuencias del cambio climático y sus efectos inciden de manera directa en la capacidad productiva de la zona y por ende a su economía; se evidencia que el cambio climático es un problema tanto meteorológico como para el desarrollo de las comunidades, porque vulnera el deterioro de los ecosistemas y los recursos que las personas necesitan para su desenvolvimiento. Consecuentemente, el desarrollo de programas y proyectos donde se involucre a la comunidad y se establezcan mecanismos y herramientas para la adaptación a dichos fenómenos es un tema que se debe trabajar desde los diversos actores sociales con presencia en el territorio.

Costa Rica cuenta con un amplio marco normativo en el tema de gestión de riesgos y adaptación al cambio climático, así como con planes que permiten la implementación de acciones concretas para cada uno de los temas abordados. Esto facilita que en el país se desarrollen diversos programas y proyectos como el de este caso de estudio; sin embargo, es necesario que el tema de gestión de riesgo y adaptación al cambio climático sea transversal en los diversos proyectos que se ejecuten en las comunidades con el fin de sensibilizar y generar acciones para adaptarse a las nuevas condiciones de vida.

El trabajo realizado desde la universidad pública con las comunidades de la CBRT ha permitido establecer mejoras sobre los ecosistemas y el bienestar de la población. Los procesos de acompañamiento desde la universidad deben mantenerse con el fin de lograr que las comunidades se apropien y logren establecer mecanismos de coordinación y/o sostenibilidad necesarios.

Las acciones ejecutadas a través del proyecto evidencian que es posible, desde las comunidades, desarrollar acciones de conservación que a su vez fomenten un uso sostenible de los ecosistemas. A través del proyecto HuriTe se evidenció que el turismo rural comunitario es una herramienta para mejorar las condiciones ambientales de los territorios y las condiciones de vida de las comunidades rurales. 


\section{Referencias}

Andrade, Isabel, y Paola Laporta. 2009. "La teoría social del riesgo. Una primera aproximación a la vulnerabilidad social de los productores agropecuarios del Sudoeste bonaerense ante eventos climáticos adversos”. Universidad Nacional de La Plata 10 (19): 1-20. https: //bit.ly/2MGTZuh.

Colmenares, Ana. 2011. "Investigación-acción participativa: una metodología integradora del conocimiento y la acción”. Voces y Silencios: Revista Latinoamericana de Educación 3 (1): 102-115. https://doi.org/10.18175/vys3.1.2012.07.

CR. 1949. Constitución política de la república de Costa Rica. Ley de iniciativa popular. Ed 34. San José: Asamblea Nacional Constituyente, 8 de noviembre.

CR Comisión Nacional de Prevención de Riesgos y Atención de Emergencias (CNE). 2015. Política nacional de gestión del riesgo 2016-2030. San José: CNE. https://bit.ly/3nOPOdL.

CR Consejo Nacional de Rectores (CONARE). 2016. Plan nacional de la educación superior universitaria estatal 2016-2020. San José: CONARE. https://bit.ly/3j1jDnK.

---. 2017. Propuesta del proyecto "Desarrollo de un modelo de gestión local integral con las poblaciones de los humedales de la cuenca baja del Río Tempisque. San José, Costa Rica”. San José: CONARE. https://bit.ly/3dI3fcN.

---. 2019. "Lineamientos para formulación, aprobación, ejecución y evaluación de proyectos y planes de trabajo financiados con recursos del fondo del sistema". San José: CONARE. https://bit.ly/319DoDI.

CR Consejo Universitario. 1987. Convenio de coordinación de la educación superior universitaria estatal en Costa Rica. Accedido febrero de 2021. https://bit.ly/37hYlze.

CR Instituto Nacional de Censos. 2002. IX Censo nacional de población: características sociales y demográficas. San José: Instituto Nacional de Estadística y Censos. https://bit. ly/2SWA1LK.

CR Ministerio de Agricultura y Ganadería. 2018. Dirección de desarrollo chorotega. Accedido enero de 2021. https://bit.ly/3rligdi.

CR Ministerio de Ambiente y Energía (MINAE). 2018a. Politica nacional de adaptación al cambio climático 2018-2030. Accedido septiembre de 2020. https://bit.ly/33Zevwb.

---.2018b. “Cambio climático ¿Cómo afecta a Costa Rica?”. Accedido septiembre de 2020. https://bit.ly/34RZEmo.

CR Ministerio de Planificación Nacional y Política Económica (MIDEPLAN). 2017. Índice de desarrollo social. Accedido septiembre de 2020. https://bit.ly/372bq0q.

---. 2019. Plan Nacional de Desarrollo y de Inversión Pública del Bicentenario 2019-2022. San José: MIDEPLAN. https://bit.ly/3nSujZD.

CR Sistema Nacional de Áreas de Conservación (SINAC). 2016. Plan de intervención ecológica de los humedales de dos áreas silvestres protegidas de la cuenca baja del Tempisque: 
Refugio Nacional de Vida Silvestre Mata Redonda y Humedal Corral de Piedra. San José: Sistema Nacional de Áreas de Conversación. https://bit.ly/3iTE7yU.

---. 2020. "Cambio climático". Accedido 15 de septiembre. https://bit.ly/3k6pppO.

Fallas, Jorge. 2011. "Ecorregiones y ecosistemas de Costa Rica: un enfoque ecosistémico". Sistema Nacional de Áreas de Conservación. Accedido octubre de 2020. https://bit.ly/3i WNDkJ.

Grupo Intergubernamental de Expertos sobre el Cambio Climático (IPCC). 2007. Resumen para responsables de políticas, en cambio climático 2007: impactos, adaptación y vulnerabilidad. Contribución del Grupo de Trabajo II al Cuarto Informe de Evaluación del Panel Intergubernamental sobre Cambio Climático. Cambridge: Cambridge University Press. https://bit.ly/2Iy54vx.

---. 2019. "Calentamiento global de $1,5{ }^{\circ} \mathrm{C}$ ". Accedido 13 de febrero de 2021. https://bit. $1 \mathrm{y} / 2 \mathrm{LS}$ BkLN.

Hartley, Marjorie. 2012. "Variabilidad climática, un análisis de la vulnerabilidad de la caficultura península". Revista Economía y Sociedad 17 (41): 65-82. https://bit.ly/31RRnpH.

Invemar, Grupo Laera, GCAP y CDKN, eds. 2014. Adaptación al cambio climático en ciudades costeras de Colombia. Guía para la formulación de planes de adaptación. Santa Marta, Invemar. https://bit.ly/36raqBL.

Murillo, Wifran, y Miriam Miranda. 2018. "Rehabilitación de ecosistemas de humedal como medidas de adaptación a los impactos de la variabilidad climática: El caso del sitio Ramsar Palo Verde, sector Catalina". Revista Ambientico 266 (8): 50-57. https://bit.ly/2SSr0TY.

Oficina de las Naciones Unidas para la Reducción del Riesgo de Desastres (UNISDR). 2009. "Terminología sobre reducción del riesgo de desastres". Ginebra: UNISDR. https://bit. ly/3b4KH3D.

---. 2015. "Marco de Sendai para la Reducción del Riesgo de Desastres 2015-2030". Nueva York: ONU. https://bit.ly/2GYy3rW.

---. 2020. “¿Qué es el Marco Sendai para la Reducción del Riesgo de Desastres?”. Accedido 15 de septiembre. https://bit.ly/2IpD0KE.

Organización de las Naciones Unidas (ONU). 1992. "Convención Marco de las Naciones Unidas sobre Cambio Climático". Nueva York: ONU. https://bit.ly/34TOqhq.

ONU. 2005. Evaluación de los ecosistemas del milenio. Nueva York: ONU.

PNUD / MIDEPLAN. 2014. Incorporación de elementos de gestión del riesgo de desastres al plan de desarrollo humano local del cantón de Nicoya 2010-2020. San José: MIDEPLAN. https://bit.ly/2FtSRXu.

Programa de las Naciones Unidas para el Desarrollo (PNUD). 2010. "Gestión del riesgo climático". Accedido septiembre de 2020. https://bit.ly/3nLrRnE.

---.2018. "Política Nacional de Humedales". Accedido febrero de 2021. http://bit.ly/3dhI $\mathrm{X} 9 \mathrm{~V}$. 
Adaptación al cambio climático y la gestión del riesgo en comunidades del río Tempisque, Costa Rica

Retana, José, y Marilyn Calvo. 2018. Riesgos ante eventos hidrometeorológicos extremos en los cantones de La Cruz, Nicoya, Hojancha y Nandayure. San José: Instituto Meteorológico Nacional. https://bit.ly/3j041RB.

Stern, Nicholas. 2006. The Economics of Climate Change. The Stern Review. Cambridge: Cambridge University Press. https://doi.org/10.1017/CBO9780511817434.

Universidad Nacional de Costa Rica. 2018. Diagnóstico situacional de las comunidades de Rosario, Roblar, Puerto Humo y Corralillo. Heredia: Escuela de Planificación y Promoción Social de la Universidad Nacional de Costa Rica.

Vargas, María, y William Gómez. 2017. "Receptividad de los ganaderos aledaños a los ecosistemas de humedal en el establecimiento y manejo de fincas pecuarias integradas". Revista Universidad en Diálogo (7): 11-23. https://doi.org/10.15359/udre.7-2.1.

Villalobos, Luis Ricardo. 2019. Enfoques y diseños de investigación social: cuantitativos, cualitativos y mixtos. San José: EUNED. 